Please do not remove this page

RMIT

UNIVERSITY

\title{
A frequency-selective mobile radio channel with hyperbolically distributed scatterers
}

Shang, Lei; Hussain, Zahir

https://researchrepository.rmit.edu.au/esploro/outputs/9921858648301341/filesAndLinks?institution=61RMIT_INST\&index=null

Shang, L., \& Hussain, Z. (2005). A frequency-selective mobile radio channel with hyperbolically distributed scatterers. Proceedings of the TENCON 2005IEEE Region 10 Conference, 1-4.

https://doi.org/10.1109/TENCON.2005.300903

Published Version: https://doi.org/10.1109/TENCON.2005.300903

Repository homepage: https://researchrepository.rmit.edu.au

(c) 2005 IEEE. Personal use of this material is permitted. However, permission to reprint/republish this material for advertising or promotional purposes or for creating new collective works for resale or redistribution to servers or lists, or to reuse any copyrighted component of this work in other works must be obtained from the IEEE.

Downloaded On 2023/04/26 23:26:46 +1000 


\title{
A FREQUENCY-SELECTIVE MOBILE RADIO CHANNEL WITH HYPERBOLICALLY DISTRIBUTED SCATTERERS
}

\author{
Lei Shang and Zahir M. Hussain, SMIEEE \\ School of Electrical and Computer Engineering \\ RMIT University, Melbourne, Victoria 3000, Australia \\ Emails: s9612136@student.rmit.edu.au,zmhussain@ieee.org
}

\begin{abstract}
In this paper we construct a frequency-selective geometrical and time-variant wireless channel model with hyperbolically distributed scatterers for a macrocell mobile environment. We verify this model against the statistical properties such as the level-crossing rate $(L C R)$, the average duration of fades $(A D F)$, the probability density function (PDF), the cumulative distribution function $(C D F)$, and the autocorrelation functions $(A C F)$ of this model. Simulation results are verified against the analytical Wide Sense Stationary Uncorrelated Scattering (WSSUS) channel model.
\end{abstract}

\section{INTRODUCTION}

All relevant components of a mobile radio system, from digital modulation techniques over channel coding through to network aspects, are determined by the propagation characteristics of the channel. Therefore, a precise knowledge of mobile radio channels is crucial for the development, evaluation and test of current and future mobile radio communication systems [1].

From the designers's viewpoint, simulation models for fading channels are extremely important for the development, performance analysis, and test of modern wireless communication systems. The designed fading channel simulator should fit the desired statistical behavior with high precision before analyzing the performance of a new mobile communication system. Accuracy, efficiency, flexibility and ease of implementation are the challenging requirements in designing the simulation models [2].

The characteristic quantities describing the statistics of mobile fading channels are the probability density function (PDF), cumulative distribution function (CDF), the autocorrelation function (ACF), the levelcrossing rate (LCR) and the average duration of fades (ADF). The level-crossing rate (LCR) and average duration of fades (ADF) are useful for designing error control codes and diversity schemes to be used in mobile communication systems, since it becomes possible to relate the time rate of change of the received signal to the signal level and velocity of the mobile [3].

In [7], a space-time geometrical based hyperbolically distributed scatterers (GBHDS) model for a macrocell mobile environment was proposed. The combination of stochastic and geometrical assumptions results in a mathematically tractable and com- putationally efficient channel model. This model provides the power of each path, the time-of-arrival (TOA), and the direction-of-arrival (DOA) of the multipath component as well as the fading effect. The model enables the simulation of downlink beamforming as well as space diversity concepts and handles both spatially narrowband and wideband signals.

Verification of the statistical properties for this newly proposed GBHDS model against the analytic fading channel model is given in [6]. The aim of this work is to extend previous results and to construct a frequency-selective GBHDS mobile channel based on previous work [6] [7] [8] [9] [10].

We first describe in Section II the stochastic reference model for the time-variant frequency-selective channel channel. In Section III, we briefly present the GBHDS model. Section IV presents the simulation results of the GBHDS frequency-selective channel simulator. The simulation results are compared with analytic results.

\section{DESCRIPTION OF THE ANALYTICAL MODEL}

For digital communication systems, propagation delay differences from multipath cannot be ignored when the transmission data rate becomes higher or symbol period becomes shorter. A multipath channel with differential path delays introduces amplitude and phase distortion is classified as frequency-selective fading channel.

The baseband input signal $s_{l}(t)$ and the output signal $r_{l}(t)$ of a time-variant frequency-selective fading channel can be expressed as [11] [12]

$$
r_{l}(t)=\sum_{n=-\infty}^{\infty} c_{n}(t) s_{l}(t-n / W)
$$

where $W$ is the bandwidth covered by the band-pass signal. Therefor, the time-variant frequency-selective channel can be modelled or represented as a tapped delay line with tap spacing $1 / W$ and tap weight coefficients $\left\{c_{n}(t)\right\}$, Hence, with the truncation by multipath spread $T_{m}$, the time-varying impulse response for the channel can be deduced from Eq. (1) as

$$
c(\tau ; t)=\sum_{n=1}^{L} c_{n}(t) \delta(\tau-n / W)
$$



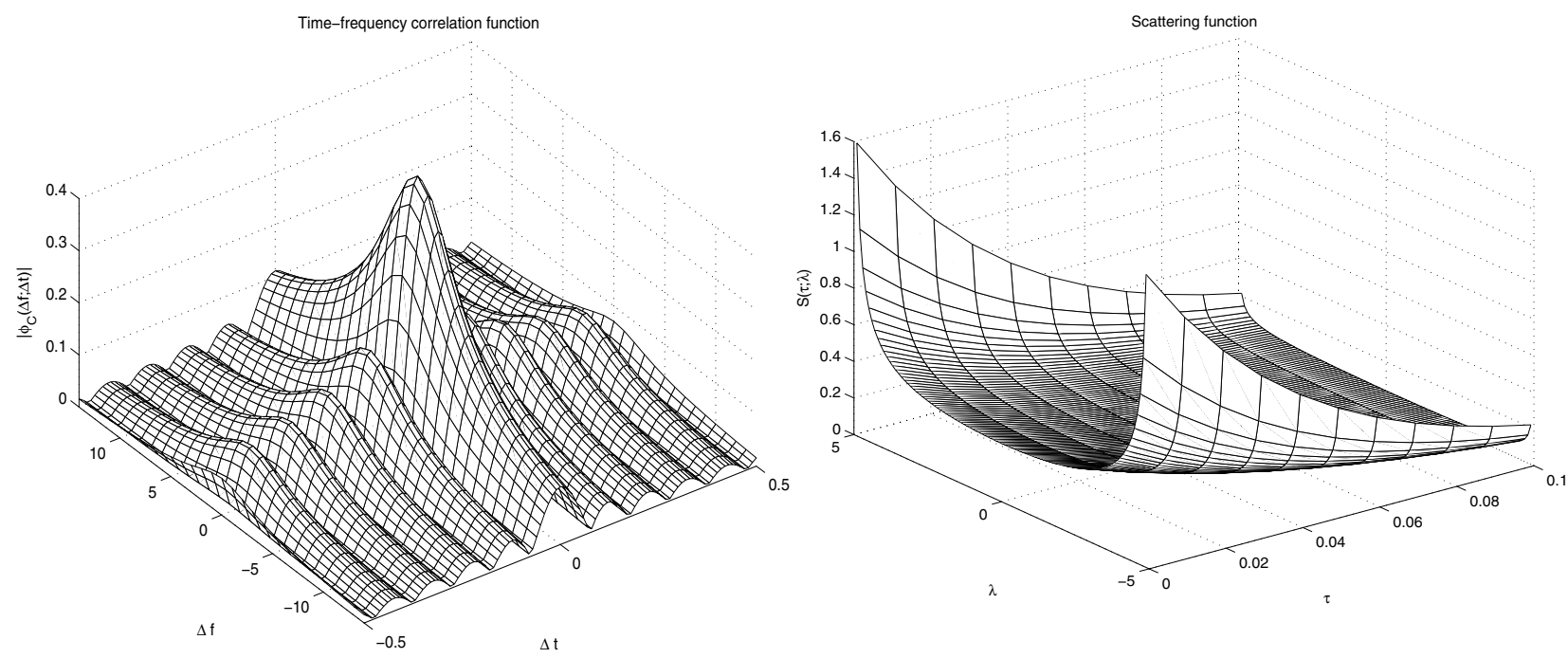

Fig. 1. Time-frequency correlation function $\left|\phi_{C}(\Delta f ; \Delta t)\right|$ $\sigma_{0}^{2}=0.5, \tau_{0}=0.05 s$, and $f_{m}=5 \mathrm{~Hz}$.

where $L=\left\lfloor T_{m} W\right\rfloor+1$ is the number of taps, and $c_{n}(t)$ and $\tau_{n}=n / W$ are the complex gains and path delays associated with the taps.

The time-variant tap weights $\left\{c_{n}(t)\right\}$ are complexvalued stationary random processes with Doppler power spectrum $S_{c}(\lambda)$. There are four types of $S_{c}(\lambda)$ specified by COST207 for different path delays [1] [12]. One of the four types, Jakes or Classical, has been investigated in a previous work [6]. The Jakes power spectral density only occurs in the case of very short propagation delays $(\leq 0.5 \mu \mathrm{s})$. The power spectral density of the taps for longer delays may be modelled as GAUS1 or GAUS2 (Sum of two Gaussian functions). The power spectral density for shortest path is modelled as Rice.

We further assume a Wide Sense Stationary Uncorrelated Scattering (WSSUS) channel introduced by [5] Due to the simplicity of WSSUS channel models, they are of great practical importance and are nowadays almost exclusively employed for modelling frequencyselective mobile radio channels [1]. For the WSSUS channel, the time-frequency correlation function $\phi_{C}(\Delta f ; \Delta t)$ or the scattering function $S(\tau ; \lambda)$ solely characterizes its statistical properties. Another common assumption is separability of scattering function. We investigate a mobile radio channel model with separable scattering function to facilitate the analysis and simulation and to develop some intuitions.

Assume a separable scattering function defined as:

$$
S(\tau ; \lambda)=\frac{1}{\sigma_{0}^{2}} S_{c}(\lambda) \phi_{C}(\tau)
$$

where $\sigma_{0}^{2}=\phi_{C}(0 ; 0)$. Then the multipath intensity profile is exponential,

$$
\phi_{C}(\tau)= \begin{cases}\frac{\sigma_{0}^{2}}{\tau_{0}} e^{-\frac{\tau}{\tau_{0}}}, & \tau \geq 0, \\ 0, & \tau<0 .\end{cases}
$$

Fig. 2. Scattering function $S(\tau ; \lambda)$ with $\sigma_{0}^{2}=0.5, \tau_{0}=$ $0.05 \mathrm{~s}$, and $f_{m}=5 \mathrm{~Hz}$.

and the Doppler power spectrum is given by

$$
S_{c}(\lambda)= \begin{cases}\frac{\sigma_{0}^{2}}{\pi f_{m} \sqrt{1-\left(f / f_{m}\right)^{2}}}, & |f| \leq f_{m}, \\ 0, & |f|>f_{m} .\end{cases}
$$

The associated time-frequency correlation function $\phi_{C}(\Delta f ; \Delta t)$ can be determined by the double Fourier transform of $S_{c}(\tau ; \lambda)$

$$
\begin{aligned}
\phi_{C}(\Delta f ; \Delta t) & =\iint S(\tau ; \lambda) e^{-j 2 \pi \Delta f \tau} e^{j 2 \pi \Delta t \lambda} d \tau d \lambda \\
& =\frac{\sigma_{0}^{2}}{1+j 2 \pi \Delta f \tau_{0}} \cdot J_{0}\left(2 \pi f_{m} \Delta t\right)
\end{aligned}
$$

For the purpose of demonstration, we let $\sigma_{0}^{2}=0.5$, $\tau_{0}=0.05$, and $f_{m}=5 \mathrm{~Hz}$, and plot the absolute value of time-frequency correlation function $\left|\phi_{C}(\Delta f ; \Delta t)\right|$ and the scattering function $S_{c}(\tau ; \lambda)$ as shown in Fig. 1 and Fig. 2, respectively. The significance of scattering function or time-frequency correlation function is that important parameters such as the multipath spread $T_{m}$ can be derived.

The above discussion will serve as the basic principle of our simulation for the frequency-selective mobile channels. In simulation, we take non-ideality into account. For example, we may use the multipath intensify profile (or power delay profile) of the typical urban defined (discrete in value and approximately exponential) by COST207 instead of Eq. (4), and the Doppler power spectrum may be a combination of the four types by COST207 mentioned previously.

\section{THE GBHDS CHANNEL MODEL}

In this section we provide a general description for the space-time geometrical-based hyperbolically distributed scatterers (GBHDS) model [7]. This model combined a scalar stochastic fading model for the local scatterers with the geometrical hyperbolic model 


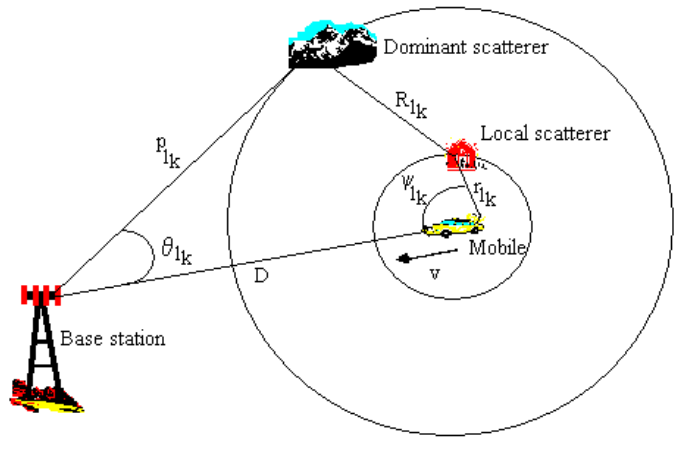

Fig. 3. Geometry of the space-time hyperbolic model.

proposed in $[8,9]$ for the distribution of the dominant scatterers. The model in [7] assumes that the scatterers are arranged circularly around the mobile, with the distances between 1) the mobile and the local scatterers and 2) the local and dominant scatterers, both being distributed hyperbolically according to an inversecosh-squared distribution. This model provides directional information as well as concerning with mobility issue. Fig. 2 shows the geometry for the GBHDS model. The angle of departure $\psi_{l_{k}}$ is uniformly distributed in the interval $[0,2 \pi]$. The angle $\theta_{l_{k}}$ is the direction of arrival at the base station, while $D$ denotes the distance between the base station and the mobile station. The mobile is located at the origin. This model has the following assumptions [7]

- The scatterers were arranged circularly around the mobile, with the distance between the mobile and the local scatterers $r_{l_{k}}$ and the distance between the local and dominant scatterers $R_{l_{k}}$ are both distributed hyperbolically.

- Signals received at the base station are plane waves propagating along the horizon ( there is no vertical component to the signal propagation).

- Scatterers are omnidirectional re-radiating elements.

- The scatterers have identical scattering coefficients.

- The macrocell antenna heights are relatively high and there is no signal scattering from locations near the base station.

The probability density functions (pdf) of the distances $r_{l_{k}}$ and $R_{l_{k}}$ for the $\mathrm{k}^{\text {th }}$ user in multiuser environment are given by [7]

$$
\begin{gathered}
f_{r_{l_{k}}}\left(r_{l_{k}}\right)=\frac{a_{1}}{\tanh \left(a_{1} R_{l s}\right) \cosh ^{2}\left(a_{1} r_{l_{k}}\right)} \\
0 \leq r_{l_{k}} \leq R_{l s}
\end{gathered}
$$

and

$$
\begin{gathered}
f_{R_{l_{k}}}\left(R_{l_{k}}\right)=\frac{a_{2}}{\tanh \left(a_{2} R_{d s}\right) \cosh ^{2}\left(a_{2} R_{l_{k}}\right)} \\
0 \leq R_{l_{k}} \leq R_{d s}
\end{gathered}
$$

where $R_{l s}$ is the radius of the circle enclosing the local scatterers, and $R_{d s}$ is the radius of the dominant scatterers circle. The applicable values of $a_{1}$ and $a_{2}$ lie in the interval $(0,1)$. From the spatial probability density functions of the scatterers in (7) and (8) we can determine the the DOA, TOA, and the signal amplitude. A comprehensive study of these models (at theoretical and simulation levels) as well as their validation with practical data have been considered. They proved to be more realistic than other models in the literature when tested against practical data $[9,10]$.

\section{A FREQUENCY-SELECTIVE GBHDS CHANNEL MODEL}

Let $T_{\text {sym }}$ be the transmission symbol rate. If

$$
T_{m} \ll T_{\text {sym }}
$$

holds, then the frequency-selectiveness or time dispersion as an effect of propagation delay differences can be ignored.

Based on the discussion in the previous sections, we develop a frequency-selective GBHDS mobile channel model. We simulate the Typical Urban (TU) (nonhilly) channel defined by COST207 [12] with all the time-variant taps $\left\{c_{n}(t)\right\}$ experience Doppler spectrum generated from GBHDS model. The simulation can be readily extended to a combination of taps with different types of Doppler spectra.

The power delay profile simulated is shown as in the following table:

\begin{tabular}{|c|c|c|}
\hline $\begin{array}{c}\text { Delay } \\
\mu s\end{array}$ & $\begin{array}{c}\text { Fractional } \\
\text { Power }\end{array}$ & $\begin{array}{c}\text { Doppler } \\
\text { Category }\end{array}$ \\
\hline 0.0 & 0.092 & Jakes \\
0.1 & 0.115 & Jakes \\
0.3 & 0.231 & Jakes \\
0.5 & 0.127 & Jakes \\
0.8 & 0.115 & Jakes \\
1.1 & 0.074 & Jakes \\
1.3 & 0.046 & Jakes \\
1.7 & 0.074 & Jakes \\
2.3 & 0.051 & Jakes \\
3.1 & 0.032 & Jakes \\
3.2 & 0.018 & Jakes \\
5.0 & 0.025 & Jakes \\
\hline
\end{tabular}

The simulation parameters for the GBHDS model are given as follows: the distance between mobile and scatterer is $1000 \mathrm{~m}$, the light seed is $3 \times 10^{8} \mathrm{~m} / \mathrm{s}$, the carrier frequency is $900 \mathrm{MHz}$, the maximum Doppler frequency is $90 \mathrm{~Hz}$.

The statistical properties of the time-variant tap weights $\left\{c_{n}(t)\right\}$ are verified according to the methods in [6] which are the probability density function, the cumulative distribution function, the autocorrelation function, the level crossing rate (LCR) and average duration of fades (ADF). 

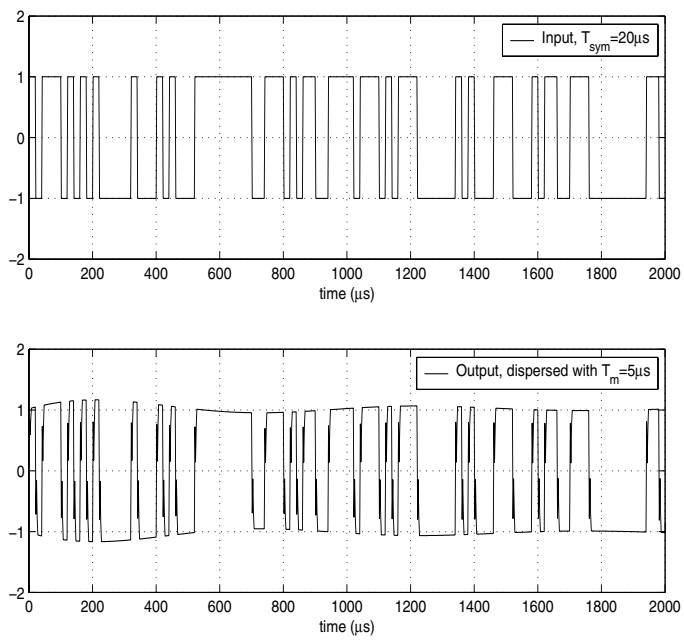

Fig. 4. BPSK of symbol period $T_{s y s}=20 \mu s$ dispersed by channel with multipath spread $T_{m}=5 \mu \mathrm{s}$.
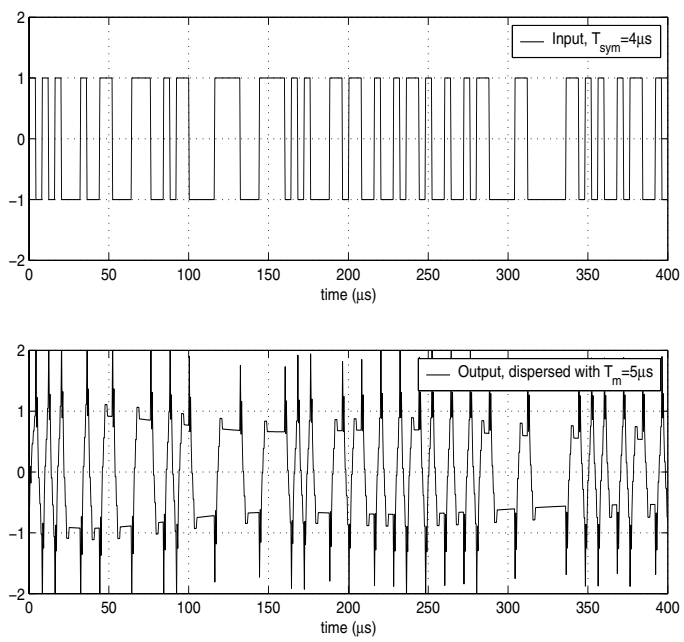

Fig. 5. BPSK of symbol period $T_{\text {sys }}=4 \mu s$ dispersed by channel with multipath spread $T_{m}=5 \mu \mathrm{s}$.

Fig. 4 shows the input BPSK signal and the output BPSK signal with $T_{m}=5 \mu \mathrm{s}$ and $T_{s y m}=20 \mu \mathrm{s}$. In this case of $T_{m} \ll T_{\text {sym }}$, the time dispersion caused by the frequency-selective GBHDS mobile channel is negligible. As shown in Fig. 5, with $T_{m}=5 \mu s$ and $T_{\text {sym }}=4 \mu \mathrm{s}$, the time dispersion by the frequencyselective GBHDS mobile channel can no longer be ignored as the channel introduces amplitude and phase distortion into the signal.

\section{CONCLUSION}

In this paper, we simulate a frequency-selective GBHDS channel. The simulated model is verified against analytical model. Important statistical characteristics such as amplitude and phase probability density function (pdf), also the higher-order statistics such as the level crossing rate (LCR) and the average duration of fades (ADF) are verified when constructing the frequency-selective GBHDS channel. The results pro- vide more support to the GBHDS model and will be useful for simulating other practical channels, such as MIMO channels, and space-time-selective mobile fading channels.

\section{REFERENCES}

[1] M. Patzold, Mobile Fading Channels, John Wiley and Sons, 2002.

[2] M. Patzold and F. Laue, "Level-Crossing Rate and Average Duration of Fades of Deterministic Simulation Models for Rice Fading Channels," IEEE Trans. Vehicular Technology, vol. 48, no. 4, pp. 1121-1129, July. 1999.

[3] T. S. Rappaport, Wireless Communications, Principles and Practice, Prentice Hall, 2002, 2nd ed.

[4] R. H. Clarke, "A Statistical Theory of Mobile-Radio Reception,” Bell Systems Technical Journal, vol. 47, pp. 957-1000, 1968.

[5] P. A. Bello, "Characterization of randomly timevariant linear channels," IEEE Trans. Comm. Syst., vol. 11, no. 4, pp. 360393, Dec. 1963.

[6] Lei Shang, Seedahmed S. Mahmoud, and Zahir M. Hussain "LeveL-Crossing Rate and Average Duration of Fades for Mobile Radio channel with Hyperbolically Distributed Scatterers," submitted to APCC2005.

[7] Seedahmed S. Mahmoud, Zahir M. Hussain, and Peter O'Shea, "Space-time model for mobile radio channel with hyperbolically distributed scatterers," IEEE Antennas and Wireless Propagation Letters, vol. 1, no. 12, pp. 211-214, 2002.

[8] _ - "Geometrical model for mobile radio channel with hyperbolically distributed scatterers," The 8th IEEE International Conference on Communications Systems, vol. 1, pp. 17-20, Singapore, Nov. 2002.

[9] — , "A Geometrical-Based Channel Model with Hyperbolically Distributed Scatterers for a Macrocell Mobile Environment with Antenna Array," Multimedia Cyberscape Journal, vol. 2, pp.1-10, 2004.

[10] —, "Spatial and Temporal Statistics for the Geometrical-Based Hyperbolic Macrocell Channel Model," Submitted to IEEE Transactions on Vehicular Technology.

[11] J. G. Proakis, Digital Communications, McGraw-Hill, 2001, 4th ed.

[12] G. L. Stuber, Principles of Mobile Communication, Kluwer Academic Publishers, 2001. 